\title{
INTENSIVE QUASI-MONOCHROMATIC, DIRECTED X-RAY RADIATION OF PLANAR CHANNELED POSITRON BUNCH.
}

\author{
Lekdar Gevorgian \\ Yerevan Physics Institute, Alikhanyan Brothers Str. 2, Yerevan 375039, Armenia
}

\begin{abstract}
The radiation of a positron in a planar channel of a crystal is studied, considering the dependence of the medium polarization on the transverse coordinate. The expression for the spectral distribution of the number of radiated photons is obtained for a single positron. It is shown that because of the medium polarization in the soft and hard regions not all positrons make a contribution to the radiation. The analytic formula for the total spectral distribution of the number of photons is obtained for the whole radiation frequency range. The theory is compared with experimental results at the hard frequency region. At the soft radiation region the number of radiated photons is of the same order as it is on the hard radiation frequency region.
\end{abstract}

Keywords: channeling, positron bunch, hard and X-Ray radiation

\section{INTRODUCTION}

In 1979 in SLAC an experimental research of radiation at planar and axial channeling of 4-16 GeV positrons was conducted with a high accuracy [1]. The spectral distribution of the radiation of the $4 \mathrm{GeV}$ positrons, channeled in planes (110) of diamond single crystal which is provided by R. O. Avakian, has a significant increase at the soft region of frequencies. This phenomenon aroused an interest because, it was related to the medium polarization influence on the radiation formation at positrons channeling.

In this paper the spectral distribution of the total radiation intensity of positrons bunch is obtained taking into account the different frequency ranges of positrons radiation. The influence of the medium polarization on the radiation spectra is investigated. A comparison of the theoretical distributions with the experiment is performed and it is shown that for satisfactory fit it is necessary to take into account the strong polarization of the atoms electron clouds through which the positrons making the main contribution in the radiation pass.

\section{THE TRAJECTORIES OF THE BUNCH POSITRONS AT PLANAR CHANNELING}

Let us choose the $y$ axis perpendicular to the chosen planes of channeling and the $z$ axis between these planes and directed along the bunch motion projection on the chosen planes. The energy of positron bunch is $E=m c^{2} \gamma$, the incidence angle relative to $z$ axis is zero and the angular divergence is sufficiently small. For the averaged potential we choose the parabolic model potential which well describes the planar channeling of positrons [2]. In this field positrons of the bunch will perform sinusoidal oscillations:

$$
s(t)=s \cos \left(\Omega_{C h} t\right), \quad s=2 y_{0} / d,
$$


where $y_{0}$ is the initial transverse coordinate, $d$ is the interplanar spacing, $\Omega_{C h}=\Omega_{0} / \sqrt{\gamma}$ is the frequency of the positron oscillations, $\Omega_{0}$ is the eigenfrequency of the planar channel:

$$
\Omega_{0}=2 c \sqrt{2 v} / d, \quad v=U_{0} / m c^{2},
$$

here $U_{0}$ is the potential well depth.

Obviously, for the channeled positrons the amplitudes have range $0<s<1$.

As the maximal amplitude is $d / 2$ for the maximal incidence angle $\vartheta_{\mathrm{L}}$ (Lindhard angle) when the positron could still be channeled we derive:

$$
\vartheta_{L} \simeq \sqrt{\frac{2 v}{\gamma}}
$$

Note that with the growth of the positron energy the oscillation frequency of the planar channeled positron is getting lower i.e. the spatial period is getting greater:

$$
l_{C h}=l_{0} \sqrt{\gamma}, \quad l_{0}=\pi d / \sqrt{2 v} .
$$

But the amplitude of oscillations as well as the radiation intensity (proportional to the square of the oscillations amplitude) depends on the initial transverse coordinate of positron. Therefore the contribution of the bunch positrons in the total radiation is different.

\section{THE SPECTRAL DISTRIBUTION OF CHANNELED POSITRONS RADIATION}

The amplitude of sinusoidal trajectory of the channeled positron is in a proportion to the maximal deviation angle of the trajectory from the $z$ axis:

$$
\vartheta_{\max }(s)=\pi d s / l_{c h}=s \sqrt{2 v / \gamma} \text {. }
$$

Then for the parameter of oscillations in channel we have:

$$
q(s)=\gamma \vartheta_{\max }(s)=s \sqrt{2 v \gamma} .
$$

The dynamics of the channeled charged particle satisfies to the dipole approximation condition $q(s) \leq 1$ if:

$$
\max (q(s)) \leq \sqrt{2 v \gamma} \leq 1
$$

In the problems of the oscillator radiation it is allowable to average the longitudinal oscillations and consider the oscillator with average constant longitudinal velocity. Because of the transversal oscillations the longitudinal Lorentz factor $\gamma_{\|}$gets smaller:

$$
\gamma_{\|}(s)=\gamma / \sqrt{Q(s)}, \quad Q(s)=1+q^{2}(s) / 2=1+v \gamma s^{2}
$$

If the motion takes place in a dispersion medium with $\rho(s)$ distribution of electrons, the dielectric permittivity in the hard frequency region is given by:

$$
\varepsilon(x)=1-\frac{\eta^{2}(s)}{4 x^{2} \gamma^{2}}, \quad x=\frac{\omega}{2 \Omega_{0} \gamma^{3 / 2}}, \quad \eta(s)=\frac{\pi r_{e}}{2} \frac{d^{2} \rho(s)}{v \gamma},
$$


where $x$ is the dimensionless frequency, $r_{e}$ is the classical electron radius, the parameter $\eta(s)$ characterize the medium polarization.

For the estimation of the average electrons density of the atoms we will use the Thomas-Fermi static model equation and the Poisson's equation for continuous electrons density

$$
\rho(s)=20 \rho_{A}(1-s)(s+a-1)^{3} / a^{4}+\rho_{0}, \quad(1-a \leq s \leq 1),
$$

where $a=2 r_{A} / d, r_{A}$ - atom radius, $\rho_{A}$ is the average electron density in atom, $\rho_{0}$ is the average electron density in crystal. In fig. 1 the model dependence of the electrons density $\rho(s)$ and polarization $\eta(s)$ in planar (110) channel of a diamond crystal is shown.

In fig. 1 the model dependence of the electrons density $\rho(s)$ and polarization $\eta(s)$ in planar (110) channel of a diamond crystal is shown.

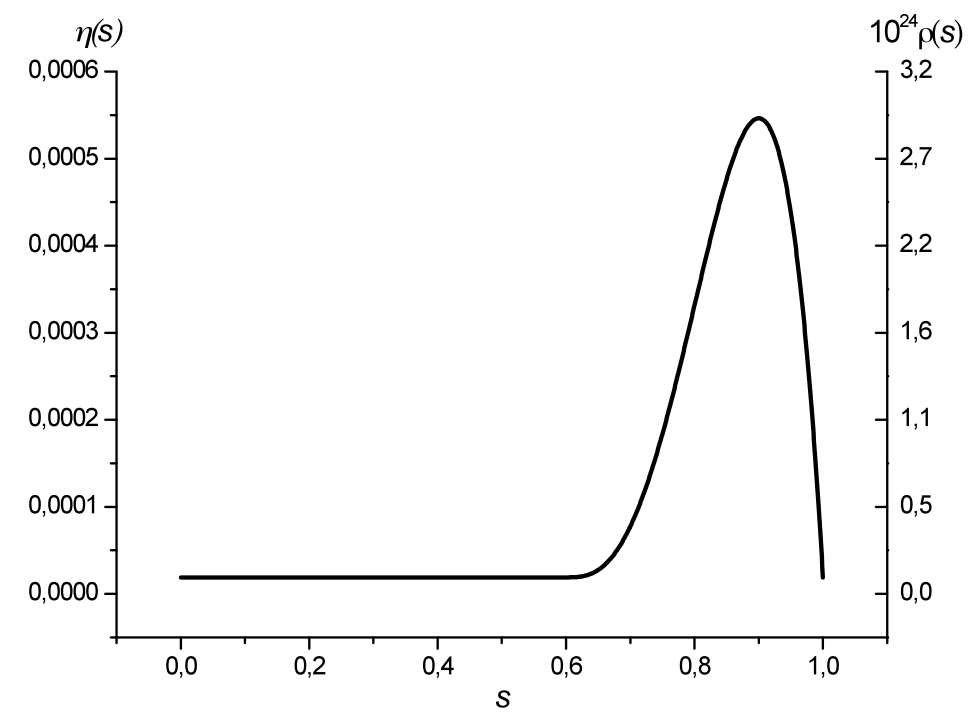

Fig. 1 The model dependence of the electrons density $\rho(s)$, and polarization $\eta(s)$ in planar channel (110) of diamond monocrystal at $E=4 \mathrm{GeV}$ positrons energy.

It is easy to show that the formulae for the frequency-angular distribution of radiation photons number by channeled relativistic positron with $s$ initial transversal coordinate in homogeneous medium [3] is also correct for the case of inhomogeneous medium:

$$
\begin{gathered}
\frac{d N}{d x d \theta^{2}}=\frac{\alpha \pi n x}{2} q^{2}(s)\left[\left(2 x \theta^{2}-1\right)^{2}+1\right] \delta\left(\theta^{2}-\phi(x)\right), \\
\phi(x)=\frac{1}{x}-Q(s)-\frac{\eta(s)}{4 x^{2}}, \quad n=\frac{L}{\pi d} \sqrt{\frac{2 v}{\gamma}} .
\end{gathered}
$$

Here $\alpha$ is the fine structure constant, $n$ is the number of positron oscillations on the length $L=n l_{c h}, \theta=\vartheta \gamma$, where $\vartheta$ is the polar angle of the radiation.

The radiation frequency range is limited by condition: $\theta^{2}=\phi(x) \geq 0$. Taking into account the weak polarization of the medium one derive: 


$$
x_{0}(s)=\eta(s) / 4 \leq x(s) \leq 1 /\left(1+v \gamma s^{2}\right)=x_{c}(s) .
$$

Then for the spectral distribution of the number of photons radiated by channeled positron with initial transverse parameter $s$ we obtain:

$$
\begin{array}{cl}
d N_{p h} / d x=\Gamma f(x, s), & \Gamma=\frac{\alpha v L}{d} \sqrt{2 v \gamma}, \\
f(x, s)=s^{2}\left(1+g^{2}(x, s)\right), & g(x, s)=2 x-1+2 v \gamma x s^{2}+\eta(s) / 2 x .
\end{array}
$$

We have extreme values for the spectrum at the following frequencies:

$$
x_{\min }^{(1)}(s)=\eta(s) / 2, \quad x_{\max }(s)=\sqrt{\eta(s) /\left(1+v \gamma s^{2}\right)} / 2, \quad x_{\min }^{(2)}(s)=1 / 2\left(1+v \gamma s^{2}\right) \text {. }
$$

The soft frequency extreme values $x_{\min }^{(1)}, x_{\max }$ depend on $\eta(s)$ and the hard frequency extreme value $x_{\min }^{(2)}(s)$ depends only on $s$.

With the increase of $s$ the number of radiated photons increases and the frequency region narrows.

\section{THE SPECTRAL DISTRIBUTION OF THE POSITRON BUNCH RADIATION}

It is clear that the transversal coordinate $s$ of a positron in a channel could get any value between 0 and 1 with the same probability.

Now the radiation frequency range is $\eta_{0} / 4=x_{0} \leq x \leq 1$, where $x_{0}=\min \eta(s) / 4$. In the interval

$$
\left(\eta_{0}+\eta_{A}\right) / 4=x_{1} \leq x \leq x_{2}=1 /(1+v \gamma)
$$

all the channeled positrons of bunch forms the radiation. Therefore in this frequency range the average spectral distribution of the radiated photons from bunch with $N_{0}$ positrons is equal

$$
\left\langle d N_{p h}^{\text {bunch }} / d x\right\rangle=\Gamma N_{0} F(x), \quad F(x)=\langle f(x, s)\rangle_{s} .
$$

At the soft frequency region the spectrum has a minimum

and a maximum

$$
x_{\min }^{(1)}=\frac{\left\langle s^{2} \eta^{2}(s)\right\rangle}{2\left\langle s^{2} \eta(s)\right\rangle}, \quad F_{\min }(x)=\frac{2}{3}-\frac{\left\langle s^{2} \eta(s)\right\rangle^{2}}{\left\langle s^{2} \eta^{2}(s)\right\rangle} .
$$

$$
x_{\max }=\frac{1}{2} \sqrt{\frac{3\left\langle s^{2} \eta(s)\right\rangle}{1+0,6 \mu}}, \quad F_{\max }(s)=\frac{2}{3}\left[1-2 \sqrt{3(1+0,6 \mu)\left\langle s^{2} \eta(s)\right\rangle}\right] .
$$

At the hard frequency range spectrum has a minimum on the frequency

$$
x_{\min }^{(2)}=\frac{1+3 \mu / 5}{2\left(1+6 \mu / 5+3 \mu^{2} / 7\right)}, \quad F_{\min } \approx 1 / 3 .
$$

The spectral distribution of the channeled relativistic positron bunch is derived for the whole frequency region (Fig. 2). 


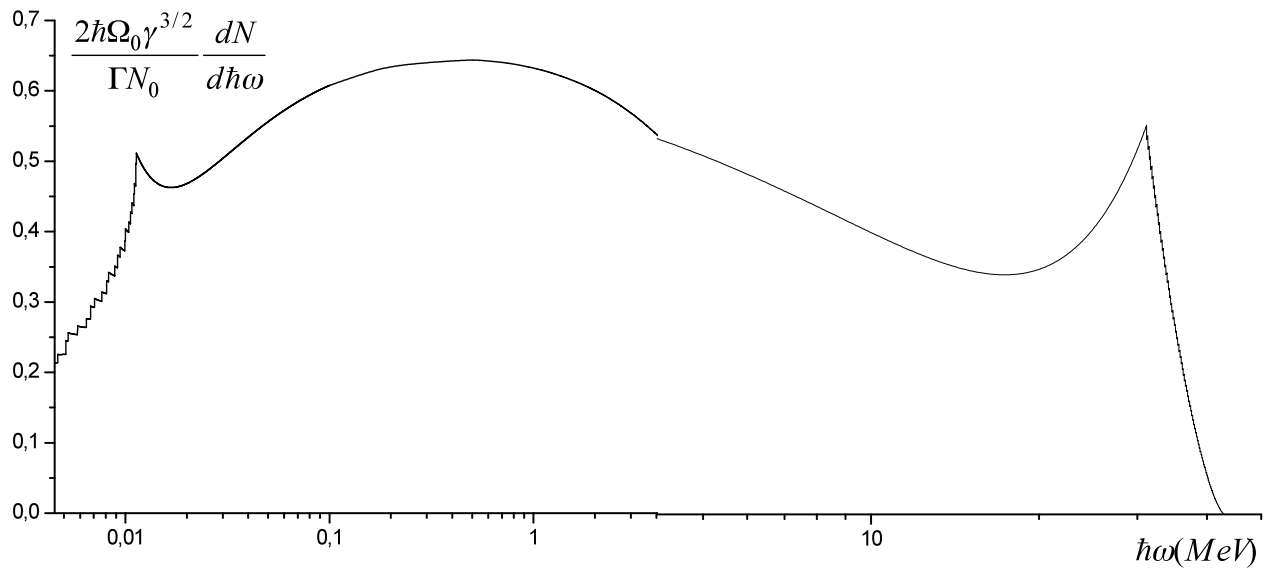

Fig. 2: The spectral distributions of the number of radiated photons by the $\Gamma N_{0}$ units for 4 GeV positron bunch channeled in the planes (110) of diamond crystal.

At the regions $\left(x_{0} ; x_{1}\right)$ and $\left(x_{2} ; 1\right)$ the spectrum decrease because of the increase of the number of radiating positrons. Thus on the frequencies $x_{1}$ and $x_{2}$ the spectrum has maximums.

At the soft frequency region the number of radiated photons is of the same order as it is at the hard region.

\section{COMPARISON WITH EXPERIMENTAL RESULTS}

The theoretical spectrum of the radiation photons number is compared with the results of experiment [1]. The considering radiation spectrum is obtained with $4 \mathrm{GeV}$ channeled positrons of the bunch parallel to the (110) planes of $80 \mu \mathrm{m}$ long diamond monocrystal. In Fig. 3 a comparison with experimental data at the hard frequency region is shown. 


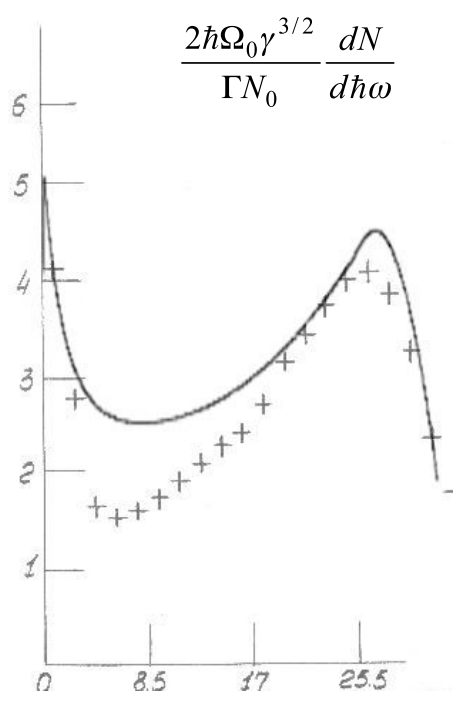

$\hbar \omega(\mathrm{MeV})$

Fig. 3. The experimental data is signed by crosses. The solid curve is the spectral distribution obtained theoretically for the number of radiated photons at $4 \mathrm{GeV}$ energy positrons channeling in the (110) planes of diamond crystal at zero angle of incidence.

\section{CONCLUSION}

The spectral distribution of the channeled charged particle radiation has the same specification as an oscillator radiation has in medium.

Channeling positrons oscillate with the same frequency but different amplitudes depending on the initial transversal coordinates. Therefore, the bunch positrons contribution in radiation is different. The radiation frequency range also depends on the initial transversal coordinate. Hence, to obtain the total spectral distribution, for a given incidence angle, an averaging by the initial coordinates of positrons is performed.

The spectral distribution of the channeled relativistic positron bunch is derived for the whole frequency region. It is shown that at the soft radiation region the number of radiated photons is of the same order as it is on the hard radiation frequency region.

The theory is compared with experiment at the high frequency region. For the coincidence of the theory with experiment on the whole frequency region it is necessary to take into account the polarization distribution in the channel.

\section{REFERENCE}

[1.] R. O. Avakyan, I. I. Miroshnichenko, J. J. Murray and T. Vigut, "Radiation of ultrarelativistic positrons moving in a crystal near crystallographic axes and planes", in Sov. Phys. JETP, 55(6), June 1982.

[2.] V. A. Bazilev, N. K. Jevago, The radiation of fast particles in medium and in external fields, M., Nauka, 1987 (in Russian).

[3.] L. A. Gevorgian, N. A. Korkhmazian, "Undulator radiation in dispersive medium", in Sov. Phys. JETP, 49(4), pp. 622-626, April 1979. 